\title{
Modelo de detección de anticuerpos neutralizantes contra IFN- $\beta$ mediante citometría de flujo
}

\author{
Juan Carlos Villa-Camacho, Juan Camilo Vargas-Zambrano, John Mario González \\ Grupo de Ciencias Básicas Médicas, Facultad de Medicina, Universidad de los Andes, Bogotá, D.C., Colombia
}

Introducción. El interferón beta (IFN- $\beta$ ) se usa para tratar la forma recaída-remisión de la esclerosis múltiple. Sin embargo, el uso de proteínas recombinantes como medicamentos puede generar la producción de anticuerpos, disminuyendo así la efectividad del tratamiento.

Objetivo. Estandarizar una técnica de detección de anticuerpos neutralizantes contra IFN- $\beta$, mediante citometría de flujo.

Materiales y métodos. Se cultivaron dos líneas tumorales humanas (U937 y K562) con IFN- $\beta 1$ a humano recombinante y mediante citometría de flujo se determinó la expresión de la proteína ISG15 intracelular. Los sueros se obtuvieron de un conejo Nueva Zelanda antes y después de la inmunización con 100.000 UI de IFN- $\beta 1$ a en adyuvante de Freund. Para la detección de anticuerpos neutralizantes, se estimularon células K562 con IFN- $\beta 1$ a preincubado con sueros de conejos a una dilución 1:20. Después de 24 horas de incubación se determinó la expresión de la proteína ISG15.

Resultados. La expresión de ISG15 fue mayor en células K562 estimuladas. La intensidad media de fluorescencia para la ISG-15 entre células en ausencia IFN- $\beta 1$ a, mostró una mediana de 198 unidades arbitrarias (UA) ( $\left.p_{25-75}=173-231 \cup A\right)$ y, en presencia de IFN- $\beta 1 a, 430 \cup A\left(p_{25-75}=316-611,5\right)$, con una diferencia estadísticamente significativa $(p=0,008)$. La presencia de anticuerpos anti-IFN- $\beta 1$ a en el suero del conejo inmunizado, hizo disminuir de forma acentuada la expresión de la ISG15 en células K562 cultivadas con IFN- $\beta 1$, en comparación con el control (mediana=3,040 UA Vs. 43,644 UA, respectivamente).

Conclusiones. Este trabajo muestra la detección de anticuerpos neutralizantes contra IFN- $\beta$ en conejos, utilizando la expresión de la proteína ISG15 por citometría de flujo.

Palabras clave: anticuerpos neutralizantes, células K562, citometría de flujo, esclerosis múltiple, interferón beta.

doi: http://dx.doi.org/10.7705/biomedica.v32i4.637

Flow cytometry model for the detection of neutralizing antibodies against of IFN- $\beta$

Introduction. Interferon beta (IFN- $\beta$ ) is a treatment for relapsing remitting multiple sclerosis. However, the therapeutic use of recombinant proteins induces a humoral immunologic response resulting in the induction of binding (BAb) or neutralizing (NAb) antibodies against the biological product. The presence of neutralizing antibodies has been associated with decreased IFN- $\beta$ treatment efficacy.

Materials and methods. Two tumor cell lines (K562 and U937) were cultivated with human recombinant IFN- $\beta 1$ a at different concentrations and lengths of time in order to measure the expression of intracellular ISG15, an inducible molecule in the IFN- $\beta 1$ a signaling cascade. Blood was obtained from non-immunized and IFN- $\beta 1$ a immunized (100,000 IU) New Zealand rabbits. The presence of BAb was evaluated by ELISA. For NAb detection, sera 1:20 dilution were added to the IFN- $\beta 1$ a-stimulated cell lines, and ISG15 expression was evaluated by flow cytometry.

Results. K562 cells provided the better cell line for the assay, stimulated with a dose of 1,000 IU of IFN- $\beta 1 \mathrm{a}$, and a 1:100 dilution for the primary antibody and a 1:200 dilution for the secondary antibody. ISG15 expression was compared between cells alone or cultivated with IFN- $\beta 1 \mathrm{a}$. Mean fluorescence intensity (MFI) for ISG-15 expression median was 198 arbitrary units (AU) with interquartile ranges of 173-231 AU for non-stimulated cells and $430 \mathrm{AU}$ with interquartile ranges of 316-611.5 AU for IFN- $\beta 1 \mathrm{a}$ stimulated cells $(p<0.01)$. Immunized rabbit sera decreased the expression of ISG-15 in K562 cells stimulated with IFN- $\beta 1 \mathrm{a}$, whereas non-immunized rabbit sera did not.

Conclusions. This rabbit model demonstrates that ISG15 expression evaluated with flow cytometry can be used as a detection assay for NAb.

\section{Contribución de los autores:}

Juan Carlos Villa-Camacho, Juan Camilo Vargas-Zambrano: ambos autores participaron por igual en el estudio, realizando los experimentos, analizando los datos y participando en la escritura del artículo.

John Mario González: diseñó y coordinó el estudio, analizó los datos y escribió el artículo. 
Key words: Antibodies, neutralizing; K562 cells, flow cytometry, multiple sclerosis, interferon-beta.

doi: http://dx.doi.org/10.7705/biomedica.v32i4.637

La esclerosis múltiple es una enfermedad inflamatoria crónica autoinmunitaria del sistema nervioso central, en la cual la infiltración de células inmunitarias (linfocitos y macrófagos) causa daños focales a la mielina y a los axones. Lo anterior se manifiesta clínicamente como una acumulación progresiva de inhabilidad cognitiva y motora en los individuos afectados (1). La esclerosis múltiple es una enfermedad relativamente común en la mayor parte de Europa, Estados Unidos, Canadá, Nueva Zelanda y el sur de Australia (2). En Colombia, un estudio en Bogotá mostró una prevalencia de esclerosis múltiple de 4,41 por 100.000 habitantes (3).

El interferón beta (IFN- $\beta$ ) se utiliza como tratamiento de elección para la forma recaídaremisión de la esclerosis múltiple, la presentación clínica más frecuente. Se ha demostrado que el IFN- $\beta$ disminuye la tasa de ataques y la gravedad de la enfermedad evaluada por el Expanded Disability Status Score (EDSS) de Kurtzke (4). Este efecto del IFN- $\beta$ en la enfermedad está dado por la disminución en la expresión de moléculas del complejo mayor de histocompatibilidad $(\mathrm{CMH})$ en células presentadores de antígenos, la inhibición de la producción de citocinas proinflamatorias, el aumento de citocinas reguladoras, la limitación del tránsito de células inflamatorias en el sistema nervioso central y la regulación de la expresión de enzimas como metaloproteinasas $(4,5)$.

Sin embargo, se sabe que el uso de productos recombinantes puede inducir la producción de anticuerpos (6-14). Se han descrito dos tipos de anticuerpos contra el IFN- $\beta$ terapéutico, los cuales se han clasificado como anticuerpos de unión (binding antibodies, BAb) y anticuerpos neutralizantes (neutralizing antibodies, NAb). Los BAb se unen a distintos epítopos antigénicos de esta citocina; sin embargo, no modifican su efecto clínico e incluso pueden desaparecer con el tratamiento continuo. La presencia de NAb está asociada con disminución de la actividad biológica del IFN- $\beta$, pérdida de eficacia clínica de éste y progresión de la enfermedad $(7,8,11,12)$. Las técnicas existentes para la detección de NAb presentan inconvenientes al involucrar

Correspondencia:

John Mario González, Universidad de los Andes, Carrera 1 № 18A-10, bloque Q, piso 8, Bogotá, D.C., Colombia

Teléfono: (571) 332 4282; fax: (571) 3324281

johgonza@uniandes.edu.co

Recibido: 31/01/12; aceptado:08/06/12 cultivos virales, depender del observador y no estar estandarizadas en cuanto al tipo de células y el virus usado $(13,14)$.

Nuestro objetivo fue la estandarización de una técnica de detección de anticuerpos neutralizantes contra IFN- $\beta 1$ a, mediante la cuantificación de la expresión de la molécula ISG15 (interferon stimulated gene 15) en una línea celular tumoral por citometría de flujo.

\section{Materiales y métodos}

\section{Líneas celulares, cultivo y determinación de la expresión de ISG15}

Se usaron las líneas tumorales K562 y U937 (ATCC, Manassas VA, USA), las cuales fueron mantenidas en medio RPMI completo y con suplemento de suero fetal bovino al $5 \%$ (Eurobio, Les Ulys, Francia). Ambas líneas se estimularon con IFN- $\beta 1$ 1a (Miltenyi Biotech, Bergisch Gladbach, Alemania) humano recombinante en una concentración de $1.000 \mathrm{UI} / \mathrm{ml}$, previa titulación de la citocina, y se incubaron durante 24 horas. Las células se fijaron con formaldehído al $2 \%$ libre de metanol (Polysciences Inc, Warrington, PA, USA) durante 10 minutos a $37{ }^{\circ} \mathrm{C}$. Posteriormente, se enfriaron durante un minuto en hielo y se permeabilizaron con $4,5 \mathrm{ml}$ de metanol al $100 \%$ durante 30 minutos.

Finalmente, luego de dos lavados con $3 \mathrm{ml}$ de PBS $1 \times$ y $0,5 \%$ de albumina sérica bovina (Sigma, St. Louis, MO, USA), se marcaron con anti-ISG15 1:200 (Cell Signaling Technology, Beverly MA, USA) y, como anticuerpo secundario, se usó anti-lgG de conejo FITC 1:100. (Jackson Immunoresearch, West Grove, PA, USA). La viabilidad celular se evaluó con 7-actinomicina D (BD Bioscience, San Diego, CA, USA) y la lectura se hizo en el citómetro FACS Canto II y el programa FACS Diva (BD Bioscience, San Jose, CA, USA).

Se contaron como mínimo 10.000 eventos y se obtuvo la intensidad media de fluorescencia. Como control de la fluorescencia, se utilizó un procedimiento de marcación similar con las células, pero sólo usando el anticuerpo secundario.

\section{Producción y evaluación de anticuerpos de unión contra IFN- $\beta 1$ a}

El antisuero se obtuvo al inmunizar en cuatro ocasiones un conejo macho Nueva Zelanda de seis meses con $100.000 \mathrm{UI}$ de IFN- $\beta 1 \mathrm{a}$, en mezcla 
$25 \% / 75 \%$ de adyuvante incompleto y completo de Freund (Sigma, St. Louis, MO, USA) con un volumen final de $1 \mathrm{ml}$. Se inmunizó en dos o tres puntos intradérmicos cada uno con una semana de separación y se obtuvo suero antes y después de la cuarta inmunización.

Para evaluar la presencia de anticuerpos de unión (BAb) a IFN- $\beta 1$ a, se realizó una ELISA indirecta. Brevemente, las placas Maxisorb ${ }^{\mathrm{TM}}$ (Nunc, Rochester, NY, USA) se sensibilizaron con $0,5 \mu \mathrm{g} /$ $\mathrm{ml}$ de IFN- $\beta 1$ a en PBS $1 \mathrm{x}$ toda la noche a $4{ }^{\circ} \mathrm{C}$. El bloqueo se hizo por una hora a temperatura ambiente $y$, posteriormente, se adicionaron los sueros del conejo de antes y después de la inmunización hasta 1:2048, diluidos en PBS 1x, 1 $\%$ albumina sérica bovina y $0.05 \%$ de Tween 20 (Sigma, St. Louis, MO, USA).

Los anticuerpos se revelaron usando un anti-conejo marcado con fosfatasa alcalina y $p$-nitrofenilfostato en solución de dietanolamina pH 9,6 como sustrato (Sigma, St. Louis, MO, USA). Entre cada reacción se lavó en cinco ocasiones con PBS 1x y $0,05 \%$ de Tween 20. La lectura se hizo a los 10 minutos en un espectrofotómetro Bio-Rad modelo 680 (Hercules, CA, USA) a $405 \mathrm{~nm}$. El punto de corte fue establecido de la media de los controles negativos más tres desviaciones estándar.

\section{Detección de anticuerpos neutralizantes contra IFN- $\beta 1$ 1a}

Para la detección de anticuerpos neutralizantes, se incubaron $1.000 \mathrm{UI}$ de IFN- $\beta 1$ a en presencia de sueros (de antes y después de la inmunización) de conejo en una dilución 1:20 durante una hora; esta dilución seleccionada es la usada en ensayos con suero de pacientes (7). Posteriormente, se adicionaron las muestras a las células K562 para su incubación durante 24 horas. Finalmente, se determinó la expresión de ISG15 por citometría de flujo, mediante el protocolo descrito anteriormente.

\section{Aspectos éticos}

Este proyecto fue aprobado por el Comité de Ética de la Universidad de los Andes. Los animales fueron mantenidos con comida y agua ad libitum. La sedación se hizo con ketamina (Rotexmedica, Trittau, Germany) para cada procedimiento.

\section{Análisis de los resultados}

Los datos obtenidos como porcentajes y valores de intensidad media de fluorescencia se analizaron utilizando la prueba $U$ de Mann-Whitney mediante el programa SPSS, versión 18 (IBM, Somers,
NY, USA) y se expresaron como la mediana y el rango intercuartílico. Los valores de $p<0,05$ se consideraron estadísticamente significativos.

\section{Resultados}

La línea celular K562 mostró una mayor expresión de la proteína ISG15 en respuesta al IFN- $\beta 1$, que la línea U937, lo que se estableció por un aumento en la intensidad media de fluorescencia dada por la expresión de dicha proteína (no se muestran los datos). En la figura 1 se observan dos gráficas de puntos de la citometría de flujo que muestra la selección de la ventana para las células K562 según el tamaño y la granularidad (figura $1 \mathrm{~A}$ ) y la selección de las células viables que son negativas para 7-actinomicina $D$ (figura $1 \mathrm{~B}$ ).

Se llevaron a cabo cinco experimentos comparando la expresión de ISG15 en células K562 que fueron cultivadas en presencia o ausencia de IFN- $\beta 1$ a. La mediana de la intensidad media de fluorescencia para las células control fue de 198 unidades arbitrarias (UA) $\left(p_{25-75}=173-231 \cup A\right) y$, para las células con IFN- $\beta 1 a$, de 430 UA $\left(p_{25-75}=316-611,5\right.$ UA), con una una diferencia estadísticamente significativa $(p=0,008)$. En la figura $1 \mathrm{C}$ se observa un histograma de citometría de flujo con el ejemplo de expresión de ISG15 en células cultivadas con y $\sin$ IFN- $\beta 1$.

Se evaluaron sueros de conejo no inmunizado e inmunizado para determinar la presencia de BAb mediante un ELISA indirecto. El ensayo se llevó a cabo por duplicado bajo las condiciones descritas. El suero control (antes de la inmunización) no fue reactivo contra el antígeno (IFN- $\beta 1$ a) en una dilución 1:16, la lectura a $405 \mathrm{~nm}$ fue de 0,085 para un punto de corte de 0,119 , establecido como se describió anteriormente. El suero después de la inmunización fue reactivo a una dilución mayor a 1:2048 con una densidad óptica de 0,298. Se hicieron cuatro ensayos por citometría de flujo, comparando la expresión de la proteína ISG15 en células K562 cultivadas en presencia de IFN$\beta 1 a$, preincubado con suero control obtenido antes y después de la inmunización. La mediana de expresión de ISG-15 para las células en presencia de suero control, fue 43,644 UA $\left(p_{25-75}=\right.$ 23,306-80,941). mientras que, en suero después de la inmunización, la mediana fue $3,040\left(p_{25-75}\right.$ $=700,5-17,816)$, lo que mostró una diferencia estadísticamente significativa $(p=0,043)$.

En la figura 2 se muestra el resultado de la inhibición de la expresión de ISG15 por el suero policlonal 

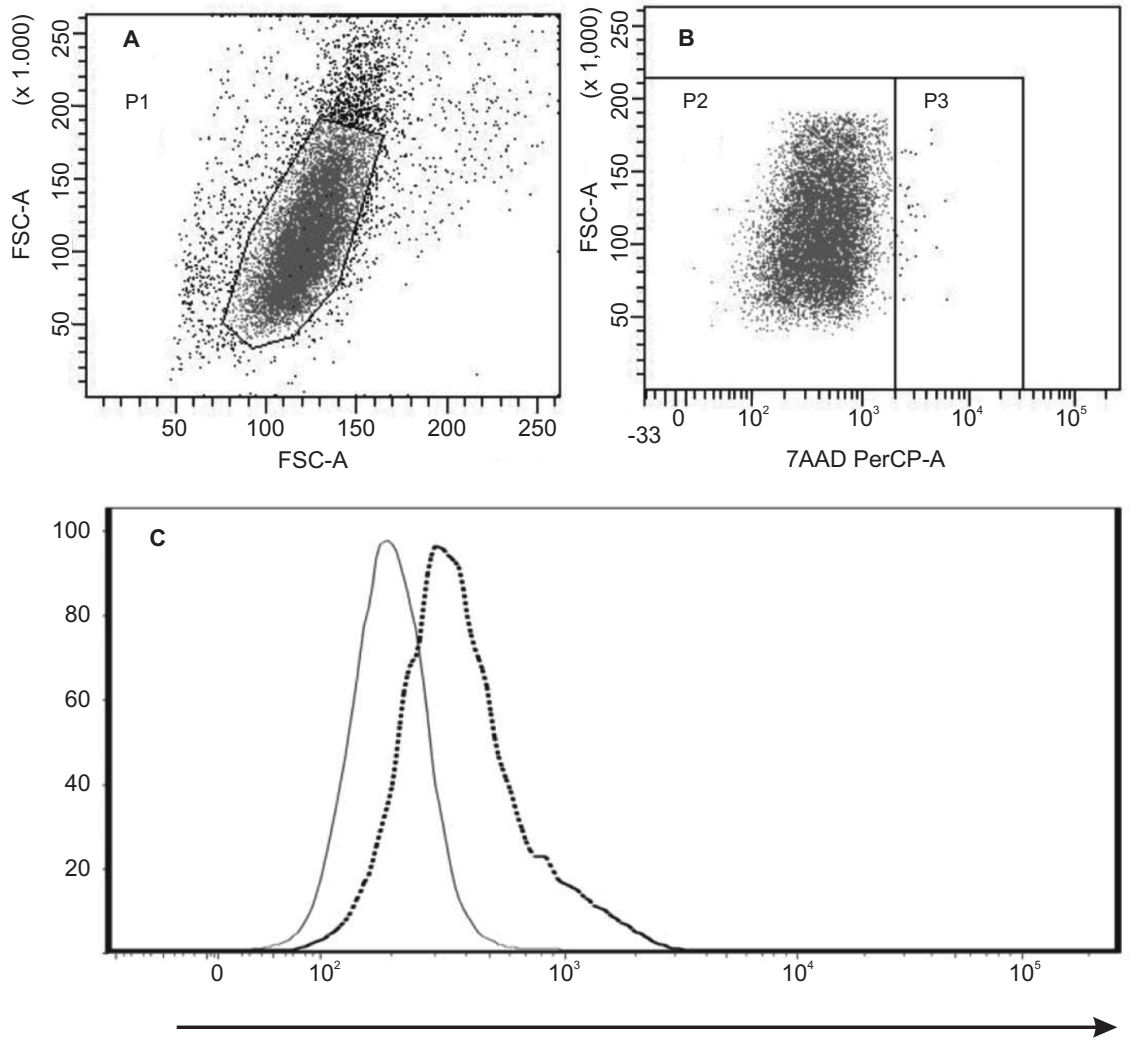

Expresión de ISG-15

Figura 1. A) Gráfica de puntos por citometría de flujo donde se observa la ventana de selección (P1) de la población de células K562 en tamaño (FSC) versus complejidad interna (SSC). B) Gráfica de puntos donde se seleccionan las células viables que son negativas para 7-actinomicina D (P2). C) Uno de cinco experimentos en un histograma por citometría de flujo donde la línea continua representa la fluorescencia para ISG15 en ausencia de estimulación con IFN- $\beta 1$ a y la línea punteada representa la fluorescencia para ISG15 en presencia de estimulación con IFN- $\beta 1$ a.

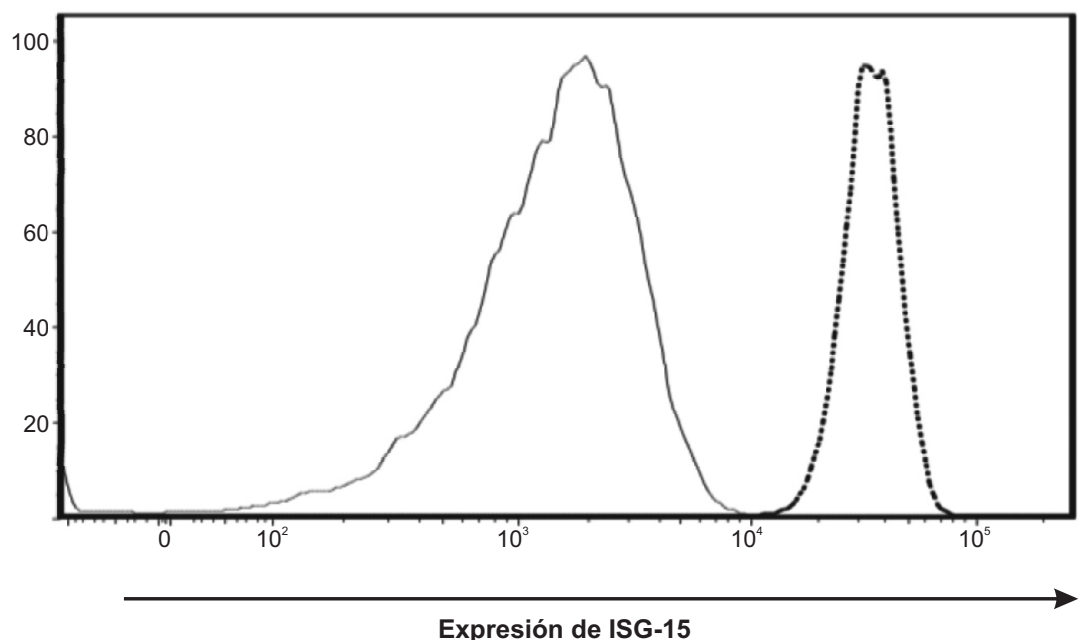

Figura 2. Ensayo de anticuerpos neutralizantes de IFN- $\beta 1$ a en suero de conejo. Se muestra un histograma obtenido por citometría de flujo en células K562 estimuladas en presencia de IFN- $\beta 1$. La línea continua es la fluorescencia de ISG15 en presencia de suero de conejo antes de la inmunización y la línea punteada la fluorescencia de ISG15 en presencia del suero de conejo después de la inmunización con IFN- $\beta 1$ a. Ensayo representativo de cuatro. 
anti-INF- $\beta 1 a$. Es de notar que, en presencia de sueros de conejos, los valores de intensidad de expresión aumentaron notablemente; esto probable se deba a la presencia de factores séricos, los cuales no interfieren con la interpretación de los resultados.

\section{Discusión}

Las células K562 expuestas a IFN- $\beta 1$ a producen significativamente más ISG15 intracelular que las células no estimuladas con este interferón, lo que muestra que la expresión de dicha proteína es inducida de forma específica por IFN- $\beta 1 a$. Usando microarreglos, en un estudio se demostró que el gen de ISG15 fue regulado positivamente en pacientes con esclerosis multiple tratados con IFN$\beta 1 b$, lo que sugiere que la proteína ISG15 podría ser usada como un biomarcador en el seguimiento del tratamiento (15).

La presencia de anticuerpos de unión (Bab) fue detectada mediante ELISA indirecta en el suero del conejo inmunizado con IFN- $\beta 1$ a y los ensayos realizados mediante citometría de flujo mostraron que efectivamente el modelo permite identificar anticuerpos neutralizantes contra IFN- $\beta 1$ a. Esto se evidencia a partir de la diferencia significativa entre los ensayos con suero control y suero policlonal anti-IFN- $\beta 1$ a.

El método propuesto representa una alternativa al ensayo citopático en cultivos celulares, pues la técnica mediante citometría no involucra el uso de cepas virales y el tiempo de desarrollo del protocolo es menor, 24 horas en citometría en comparación con 48 a 72 horas en el ensayo citopático. Además, para estudiar el efecto citopático se utilizan diferentes tipos de virus (VSV, MCMV) y diferentes tipos de líneas celulares; la lectura de los resultados se hace observando efectos citopáticos o disminución de la capacidad metabólica celular medida por MTT $(13,14)$.

Con el uso de la citometría de flujo se pueden estandarizar rápidamente la línea celular, el tipo de interferón, la dosis de estimulación, el tiempo de incubación y las diluciones de anticuerpos que se deben en el ensayo. Asimismo, este ensayo es más específico pues la expresion de la ISG15 es una medida directa de la actividad del IFN- $\beta 1$ a (16) y la interpretación no depende del operador, ya que la semicuantificación de la expresión de ISG15 se mide por el citómetro de flujo. No existen estudios comparativos del uso de ISG15 medida por citometría de flujo como marcador para determinar anticuerpos neutralizantes contra IFN$\beta 1$ a. Por lo tanto, este modelo podría ser utilizado en pacientes con esclerosis múltiple tratados con IFN- $\beta$ de acuerdo con las guías de manejo internacionales (7).

\section{Conflictos de intereses}

Los autores no reportan ningún conflicto de interés.

\section{Financiación}

Este proyecto fue financiado por la Vicerrectoría de Investigaciones de la Universidad de los Andes.

\section{Referencias}

1. Compston A, Coles A. Multiple sclerosis. Lancet. 2008;372:1502-17.

2. Ascherio A, Munger K. Epidemiology of MS: From risk factors to prevention. Semin Neurol. 2008;1:17-28.

3. Toro J, Sarmiento OL, Díaz del Castillo A, Satizabal CL, Ramírez, JD, Montenegro AC, et al. Prevalence of multiple sclerosis in Bogotá, Colombia. Neuroepidemiology. 2007;28:33-8.

4. Coyle PK. Existing therapies for multiple sclerosis offer proven efficacy and safety. Curr Opin Neurol. 2009;22:S4-9.

5. Yong VW, Chabot S, Stuve O, Williams G. Interferon beta in the treatment of multiple sclerosis: Mechanisms of action. Neurology. 1998;51:682-9.

6. Polman $\mathbf{C H}$, Bertolotto A, Deisenhammer F, Giovannoni G, Hartung HP, Hemmer B, et al. Recommendations for clinical use of data on neutralizing antibodies to interferonbeta therapy in multiple sclerosis. Lancet Neurol. 2010;9:74050 .

7. Sorensen PS, Ross C, Clemmesen KM, Bendtzen K, Frederiksen JL, Kristensen $\mathrm{O}$, el at. Clinical importance of neutralizing antibodies against interferon beta in patients with relapsing-remitting multiple sclerosis. Lancet. 2003;362:1184-91.

8. Goodin DS, Frohman EM, Hurwitz B, O'Connor PW, Oger JJ, Reger AT, et al. Neutralizing antibodies to interferon beta: Assessment of their clinical and radiographic impact. An evidence report - Report of the Therapeutics and Technology Assessment Subcommittee of the American Academy of Neurology. Neurology. 2007;68:977-84.

9. PRISMS (Prevention of Relapses and Disability by Interferon beta-1a Subcutaneously in Multiple Sclerosis) Study Group. Randomized double-blind placebo-controlled study of interferon beta-1a in relapsing/remitting multiple sclerosis. Lancet. 1998;352:1498-504.

10. PRISMS-4 Study Group. Long-term efficacy of interferonbeta-1a in relapsing MS. Neurology. 2001;56:1628-36.

11. Soelberg Sorensen PS, Koch-Henriksen N, Ross C, Clemmense KM, Danish Multiple Sclerosis Study Group. Appearance and disappearance of neutralizing antibodies during interferon-beta therapy. Neurology. 2005;65:33-9.

12. Farrell RA, Giovannoni G. Measuring and management of anti-interferon beta antibodies in subjects with multiple sclerosis. Mult Scler. 2007;13:567-77. 
13. Oliver B, Órpez T, Mayorga C. Neutralizing antibodies against IFN beta in patients with multiple sclerosis: A comparative study of two cytopathic effect tests (CPE) for their detection. J Immunol Methods. 2009;351:41-5.

14. Massart C, Gibassier J, de Seze J, Debouviere M, Moreau $\mathrm{T}$, Pelletier $\mathrm{J}$, et al.. Determination of interferon beta neutralizing antibodies in multiple sclerosis: Improvement of clinical sensitivity of a cytopathic effect assay. Clin Chim Acta. 2008;391:98-101.
15. Serrano-Fernández P, Möller S, Goertsches R, Fiedler H, Koczan D, Thiesen HJ, et al. Time course transcriptomics of IFNB1b drug therapy in multiple sclerosis. Autoimmunity. 2010;43:172-8.

16. Platanias, L. Mechanisms of type-I- and type-II-interferonmediated signaling. Nat Rev Immunol. 2005;5:375-86. 